\title{
Efecto del tipo de concentrado sobre indicadores sanguíneos del metabolismo de energía y de proteínas en vacas lecheras en pastoreo primaveral ${ }^{\#}$
}

\author{
Effects of two type of concentrate on energy and protein blood metabolites \\ in grazing dairy cows during spring \\ M Noro ${ }^{1,3}$, V Vargas ${ }^{3}$, R G Pulido ${ }^{2}$, F Wittwer ${ }^{3 *}$ \\ ${ }^{1}$ Programa Doctorado Ciencias Veterinarias, Universidad Austral de Chile, Valdivia, Chile. ${ }^{2}$ Instituto de Ciencia Animal y \\ Tecnología de Carnes. ${ }^{3}$ Instituto de Ciencias Clínicas Veterinarias, Universidad Austral de Chile, Valdivia, Chile.
}

\begin{abstract}
SUMMARY
The objective of the study was to evaluate the effect of supplementation with starch or fibre-based concentrates on blood metabolites in dairy cows fed spring pasture. Twenty seven lactating Friesian cows producing $30.0 \mathrm{~L} /$ day were assigned to completely randomized design for 42 days. The treatments included: Grazing alone (TG), grazing plus $6 \mathrm{~kg} / \mathrm{d}$ of sugar beet pulp-based concentrate (TA) and grazing plus $6 \mathrm{~kg} / \mathrm{d}$ of cereal-based concentrate (TF). The cows were supplemented twice a day and managed under a strip grazing system on pasture consisting mainly of perennial ryegrass. Six coccigeal blood samples from each cow were obtained for seven consecutive days after the morning milking in vacuum tubes containing sodium heparin and sodium fluoride. Plasma was separated after centrifugation, frozen at $-25^{\circ} \mathrm{C}$ and analyzed for $\beta \mathrm{OH}$-butyrate, glucose, urea and albumin. The unsupplemented group had a lower glycaemia and a higher $\beta \mathrm{OH}$-butyrate concentrations than $\mathrm{TA}$ and $\mathrm{TF}(\mathrm{P}<0.05)$. It was observed a prevalence of $44 \%$ sub clinical ketosis in TP group, and no cases in TA or TF. The glycaemia were higher in TA $(\mathrm{P}<0.05)$. The plasmatic urea concentrations were lower $(\mathrm{P}<0.05)$ in TS and higher in TG $(\mathrm{P}<0.05)$. Among the treatments not differences were found for the plasmatic albumin concentrations $(\mathrm{P}>0.05)$. From the results it can be concluded that supplementation with starch concentrate advantage the energetic balance in lactating dairy cows at pasture. The supplementation with concentrate based on starch or fibre decreased the risk of sub clinical ketosis, and increase the utilization of pasture nitrogen reducing plasma urea concentrations.
\end{abstract}

Palabras clave: vacas, pastoreo, suplementación, metabolismo.

Key words: cows, grazing, supplementation, metabolism.

\section{INTRODUCCION}

La pradera es el recurso más abundante y económico utilizado por los sistemas productivos lecheros y, como único alimento, es capaz de mantener altos niveles de productividad bajo condiciones de disponibilidad ad libitum (Pulido y col 1999, Pulido y col 2001). Si bien durante la primavera se concentra el $40 \%$ o más de la producción anual de pradera permanente en el sur de Chile, ésta se caracteriza por poseer un elevado contenido de proteína cruda y un bajo porcentaje de materia seca (Ruiz 1997), disminuyendo el consumo por el animal (Leaver 1985). Estas características imponen una de las principales limitantes nutricionales para alcanzar un máximo consumo de materia seca y de energía en los

Aceptado: 27.03.2006.

\# FONDECYT No 1030331

* E-mail: fwittwer@uach.cl. Fax: (63) 221457, Casilla 567, Universidad Austral de Chile (UACH). sistemas que utilizan vacas de alta producción (Peyraud y Delaby 2001). Por lo tanto, la suplementación energética es necesaria para mejorar la producción.

La principal fuente energética usada como suplemento en las explotaciones lecheras de la zona sur del país es el almidón de los granos de cereales, tales como el maíz, la avena y la cebada. Otra fuente de suplementación energética de uso menos común es la fibra digestible de la coseta de remolacha azucarera. La suplementación con concentrados ricos en almidón se caracteriza por modificar las proporciones molares de los ácidos grasos volátiles, producto de la degradación del almidón en el rumen, favoreciendo la producción de propionato, que es el principal precursor de glucosa en los rumiantes, situación que influye positivamente en el metabolismo energético (Knowlton y col 1998, Bargo y col 2003). Por su parte, la coseta de remolacha fermetaría más lentamente que el almidón en el rumen, produciendo una menor intensidad fermentativa a los microorganismos ruminales y una mayor proporción de acetato en relación al propionato, lo que favorecería la síntesis de grasa de leche (Webster 1993). 
La suplementación con concentrados energéticos, además de aumentar el consumo de materia seca en vacas en lactancia a pastoreo, mejora la eficiencia de utilización del nitrógeno de la pradera por parte de los microorganismos ruminales (Sinclair y col 2000, Bargo y col 2002), aumentando la glucemia y disminuyendo la concentración de urea en sangre, ajustando consecuentemente el metabolismo intermediario energético y proteico (Sinclair y col 2000).

Los trastornos metabólicos de origen nutricional son frecuentes en los primeros meses de lactancia y durante la primavera en vacas de alta producción a pastoreo (Wittwer y col 1987). Al inicio de la lactancia las vacas cursan con un grado de balance energético negativo, aumentando la tasa de movilización de reservas lipídicas e incrementando las concentraciones en sangre y leche de cuerpos cetónicos, como el $\beta \mathrm{OH}$-butirato, acetoacetato y acetona (Herdt 1988, Herdt 2000). En estas condiciones los animales pueden cursar con cetosis subclínica o clínica, provocando pérdidas económicas por costos de tratamientos y menor producción de leche. La cetosis subclínica es de especial interés en consideración a su elevada prevalencia, la que en vacas Holstein puede alcanzar un $43 \%$ durante la segunda semana de lactancia con pérdidas que pueden alcanzar a USD 78 por animal (Geishauser y col 2001).

La urea plasmática es un indicador sensible de la ingesta de proteína cruda y su sincronismo con la liberación de energía en el rumen, ya que sus concentraciones son dependientes de la producción y absorción del amonio ruminal (Sinclair y col 1993, Sinclair y col 2000). Así, una adecuada suplementación con concentrados, que aporte energía, mejora el aprovechamiento del amonio ruminal, disminuyendo la concentración plasmática de urea.

El presente ensayo tuvo por objeto determinar el efecto de la suplementación con concentrados a base de cebada o coseta de remolacha sobre indicadores del metabolismo energético y proteico en vacas en lactancia a pastoreo durante la primavera.

\section{MATERIAL Y METODOS}

Ubicación del ensayo. Se realizó en la estación experimental Vista Alegre de la Universidad Austral de Chile, ubicada 9 kilómetros al norte de la ciudad de Valdivia, provincia de Valdivia, X Región, Chile (3948'LS y $\left.73^{\circ} 13^{\prime} \mathrm{LO}\right)$, a una altura de 12 metros sobre el nivel del mar.

Período experimental. El ensayo tuvo una duración de 49 días, el que fue dividido en una fase inicial o preexperimental de 7 días y una fase experimental de 42 días, la que se llevó a cabo entre el 22 de septiembre y el 9 de noviembre de 2003.

Animales. Se utilizaron 27 vacas de raza Frisón Negro seleccionadas a base de producción de leche $(30 \pm 5 \mathrm{~L})$, días posparto (53 \pm 14 días), peso vivo $(538 \pm 60 \mathrm{~kg})$, condición corporal ( 2 a 3 , escala de 1 a 5 ) y número de lactancias $(3,8 \pm 1,8)$. Cada uno de los animales fue individualizado por medio del número y color del autocrotal de registro.

Tratamientos. Las vacas se distribuyeron de acuerdo a su producción de leche en tres grupos de 9 animales cada uno, en un diseño experimental aleatorio continuo, en el que los animales permanecieron en el mismo grupo durante todo el experimento. Los tratamientos fueron: solo a pastoreo (TP), pastoreo más $6 \mathrm{~kg}$ de concentrado amiláceo (TA) y pastoreo más $6 \mathrm{~kg}$ de concentrado fibroso (TF). El concentrado fue entregado a los animales suplementados en dos porciones de $3 \mathrm{~kg}$ cada una, durante las ordeñas de la mañana y de la tarde.

Dieta y manejo. Se utilizaron 8,7 hectáreas de pradera permanente mejorada, divididas en 7 potreros, los que se encontraban a aproximadamente 400 metros de distancia de la sala de ordeña. La pradera era constituida mayormente de ballica (Lolium sp.), con uniformidad en cuanto a composición botánica, edad y manejo (cuadro 1). Los tres grupos de vacas fueron manejados independientemente y se mantuvieron separados, pastoreando una franja de pradera con cambio dos veces por día. Para determinar la superficie de cada franja se utilizó el método de medición de la altura de la pradera prepastoreo y postpastoreo (Hodgson 1990). Las franjas fueron reguladas por un cerco eléctrico móvil y se ofreció una disponibilidad de pradera de aproximadamente entre 35 a $40 \mathrm{~kg}$ de MS vaca/día, entregada en dos franjas diarias, destinada a ofrecer un disponibilidad ad libitum de pradera. Las áreas de pastoreo fueron estimadas cada dos días utilizando un plato medidor de $30 \mathrm{~cm}$ de diámetro ${ }^{5}$ para determinar el volumen de la pradera comprimida caminando en el potrero en un patrón de

Cuadro 1. Composición nutricional promedio de la pradera y de los concentrados utilizados durante el experimento.

Chemical composition of the herbage and supplements offered throughout the experiment.

\begin{tabular}{lccc}
\hline & \multicolumn{2}{c}{ Concentrados } & Pradera \\
\cline { 2 - 4 } & Amiláceo $^{1}$ & Fibroso $^{2}$ & X \pm DE \\
\hline MS (\%)* & 88,1 & 88,9 & $15,6 \pm 1,4$ \\
PC (\%) & 12,1 & 11,6 & $25,1 \pm 2,3$ \\
FDN (\%) & 26,1 & 39,9 & $52,1 \pm 4,4$ \\
EE (\%) & 1,8 & 1,6 & - \\
CT (\%) & 2,5 & 6,9 & $9,3 \pm 1,7$ \\
CNE (\%) & 57,5 & 40,0 & - \\
EM & 3,1 & 3,1 & $2,7 \pm 0,1$ \\
\hline
\end{tabular}

* MS: Materia seca; PC: Proteína Cruda; FDN: Fibra detergente neutro; EE: Extracto etéreo; CT: Cenizas totales; CNE: Carbohidratos no estructurales, $\mathrm{CNE}=100-(\mathrm{FDN}(\%)+\mathrm{PC}(\%)+\mathrm{EE}(\%)+\mathrm{CT}$, EM: Energía Metabolizable (Mcal/kg MS).

${ }^{1}$ Base a cebada. ${ }^{2}$ Base a coseta de remolacha. 
W. Finalizado el pastoreo se realizaron cortes de limpieza. La mezcla mineral estuvo disponible en saleros ubicados en el potrero y en la sala de ordeña. Los concentrados utilizados fueron peletizados, isoproteicos e isocalóricos, los que se caracterizaron por poseer un mínimo de $11 \%$ PC y 3,1 Mcal/EM (cuadro 1). El concentrado amiláceo tuvo como fuente mayoritaria de carbohidratos al almidón de cebada y el concentrado fibroso a la fibra de coseta de remolacha. El agua se ofreció ad libitum en los potreros y sala de espera de ordeña.

Procedimientos y muestreos. A partir del $7^{\circ}$ día del período experimental se obtuvieron 6 muestras de sangre a todos los animales con un lapso de 7 días entre muestras. Las muestras de $7 \mathrm{ml}$ de sangre se obtuvieron mediante venopunción yugular o coccígea, de los cuales $3 \mathrm{ml}$ fueron adicionados a un tubo con $\mathrm{NaF}$ para la determinación de la glucemia y $4 \mathrm{ml}$ fueron adicionados a un tubo con heparina sódica para la determinación de las concentraciones de $\beta \mathrm{OH}$-butirato, urea y albúmina plasmática. Posteriormente, las muestras de sangre fueron centrifugadas por 10 minutos a $2.000 \mathrm{rpm}$ y el plasma fue alicuotado y congelado en microtubos de $1,5 \mathrm{ml}$.

Se determinaron las concentraciones plasmáticas de glucosa (GOD-PAP, Roche No 1448668), urea (GD UV cinético, Human, $\mathrm{N}^{\circ} 10521$ ) y albúmina (verde de bromo cresol, Human, $\mathrm{N}^{\circ}$ 10560) en un autoanalizador Cobas

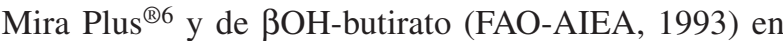
un espectrofotómetro Hitachi $4020^{\circledR}$. La prevalencia de cetosis subclínica fue analizada considerando como positivas las muestras que presentaron una concentración plasmática de $\beta \mathrm{OH}$-butirato $>1,4 \mathrm{mmol} / \mathrm{L}$ (Geishauser y col 2001, Carrier y col 2004).

Análisis estadístico. Los datos obtenidos fueron analizados empleando estadística descriptiva $(\mathrm{X} \pm \mathrm{DE})$. Se estableció normalidad de la distribución de los datos de cada variable por la prueba de Shapiro-Wilk y homocedasticidad entre los tratamientos mediante la prueba de Barttlet's. Se compararon las variables paramétricas y homocedásticas mediante ANDEVA para muestras repetidas, y prueba de comparaciones múltiples de Tukey, los datos no paramétricos o heterocedásticos fueron comparados mediante ANDEVA no paramétrico de Kruskall Wallis, empleando el programa estadístico Statistix 8.0 (NH Analytical Software, Roseville, MN, USA) con un nivel de significación de 95\%.

\section{RESULTADOS}

Las características nutritivas de la pradera y de los alimentos suplementarios se presentan en el cuadro 1 .

\footnotetext{
${ }^{5}$ Ashgrove Plate Meter, Hamilton, New Zealand.

${ }^{6}$ Roche.
}

La presión de pastoreo media fue de $37 \mathrm{~kg}$ MS/vaca al día, valor que estuvo dentro del rango objetivo (35 a 40 $\mathrm{kg}$ MS/vaca/día) para el experimento (Pulido y col 2004a , Pulido y col 2004 ${ }^{\mathrm{b}}$ ). La concentración de glucosa plasmática fue superior $(\mathrm{P}<0,05)$ en las vacas suplementadas con concentrado amiláceo (TA), comparado con los tratamientos TP y TF, los que fueron similares entre sí (P > 0,05; cuadro 2). Durante el período experimental la glucemia presentó un descenso, siendo al final del experimento inferior que al inicio en los tratamientos TA y TF $(\mathrm{P}<0,05)$, y manteniéndose constante en TP ( $P>0,05$; figura 1a).

Similar a la glucemia, la concentración plasmática de $\beta \mathrm{OH}$-butirato fue menor en las vacas suplementadas con concentrado amiláceo TA $(\mathrm{P}<0,05)$, comparado con los tratamientos TP y TF que fueron similares $(\mathrm{P}>0,05$; cuadro 2). No se observaron diferencias entre muestreos dentro de un mismo grupo $(\mathrm{P}>0,05)$.

Los animales de los grupos suplementados no presentaron cetosis subclínica, mientras que en el grupo TP cuatro vacas $(44 \%)$ cursaron con cetosis subclínica, con concentraciones medias de $\beta \mathrm{OH}$-butirato de 1,60 $\pm 0,16$ $\mathrm{mmol} / \mathrm{L}$. En dos de estas vacas se diagnosticó cetosis subclínica el día 7, una el día 35 y, en la otra, desde el día 21 hasta el día 35. Los animales con cetosis subclínica se encontraban con $80 \pm 10$ días de lactancia y producían 27,4 $\pm 2,8$ litros de leche día. El animal que presentó cetosis subclínica durante tres semanas tenía más días en lactancia (108 días) y menor producción láctea $(24$ L/d) entre los afectados.

La concentración plasmática de urea fue más elevada en las vacas que no recibieron concentrado (TP), comparado con las que fueron suplementadas, TA y TF, $(\mathrm{P}<0,05)$, y entre estas últimas fue más baja en el grupo suplementado con concentrado amiláceo, TA, entre los 21 a los 35 días del experimento ( $\mathrm{P}<0,05$; cuadro 2$)$. La concentración de urea, si bien presentó diferencias entre

Cuadro 2. Concentraciones plasmáticas de glucosa, $\beta \mathrm{OH}$-butirato, urea y albúmina (medias $\pm \mathrm{DE}^{*}$ ) en vacas en lactancia a pastoreo (TP) y suplementadas con concentrado amiláceo (TA) o fibroso (TF) durante 42 días.

Plasma concentrations of glucose, $\beta \mathrm{OH}$-butyrate, urea and albumin (mean \pm SD*) in lactating cows grazing rye grass $(\mathrm{TP})$ and supplemented with concentrate based on starch (TA) or fiber (TF) during 42 days.

\begin{tabular}{|c|c|c|c|c|}
\hline \multirow[b]{2}{*}{ Variables } & \multicolumn{3}{|c|}{ Tratamientos } & \multirow[b]{2}{*}{$P$} \\
\hline & $\mathrm{TP}(\mathrm{n}=9)$ & $\mathrm{TA}(\mathrm{n}=9)$ & $\mathrm{TF}(\mathrm{n}=9)$ & \\
\hline Glucosa (mmol/L) & $3,09 \pm 0,21^{b}$ & $3,25 \pm 0,53^{\mathrm{a}}$ & $3,09 \pm 0,25^{b}$ & 0,023 \\
\hline$\beta O H-b u t i r a t o(m m o l / L)$ & $0,86 \pm 0,36^{\mathrm{a}}$ & $0,59 \pm 0,23^{b}$ & $0,82 \pm 0,26^{\mathrm{a}}$ & 0,000 \\
\hline Urea $(\mathrm{mmol} / \mathrm{L})$ & $8,18 \pm 1,20^{\mathrm{a}}$ & $6,61 \pm 1,40^{c}$ & $7,26 \pm 1,58^{b}$ & 0,000 \\
\hline Albúmina $(\mathrm{g} / \mathrm{L})$ & $38,6 \pm 2,1^{\mathrm{a}}$ & $38,4 \pm 1,7^{\mathrm{a}}$ & $38,4 \pm 3,1^{\mathrm{a}}$ & 0,929 \\
\hline
\end{tabular}

* Valores obtenidos en 6 muestreos durante 42 días. Letras distintas en una fila señalan diferencias, $\mathrm{P}<0,05$. 
los muestreos dentro de un mismo grupo ( $\mathrm{P}<0,05)$, estas no tuvieron una tendencia definida, observándose valores más elevados a los días 7 y 28, e inferiores a los 21 y 42 (figura 1 b).

Las concentraciones de albúmina plasmática fueron similares en los tres tratamientos y constante durante el período experimental ( $\mathrm{P}>0,05$; cuadro 2).

\section{DISCUSION}

Las concentraciones plasmáticas de glucosa en los tres grupos se mantuvieron dentro de los rangos de referencia (2,5 a 4,16 mmol/L), concordante con los antecedentes que indican que la glucemia de los bovinos se mantiene dentro de los rangos fisiológicos con leves variaciones estacionales, producto del fuerte mecanismo de control homeostático hormonal (Payne y Payne 1987). A su vez, las vacas del grupo TA presentaron una glucemia más alta $(\mathrm{P}<0,05)$ que las de los tratamientos TP y TF,

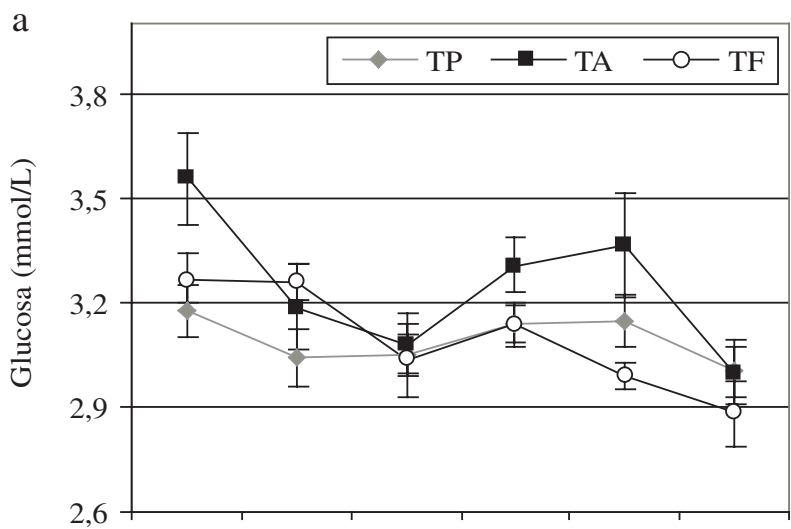

b

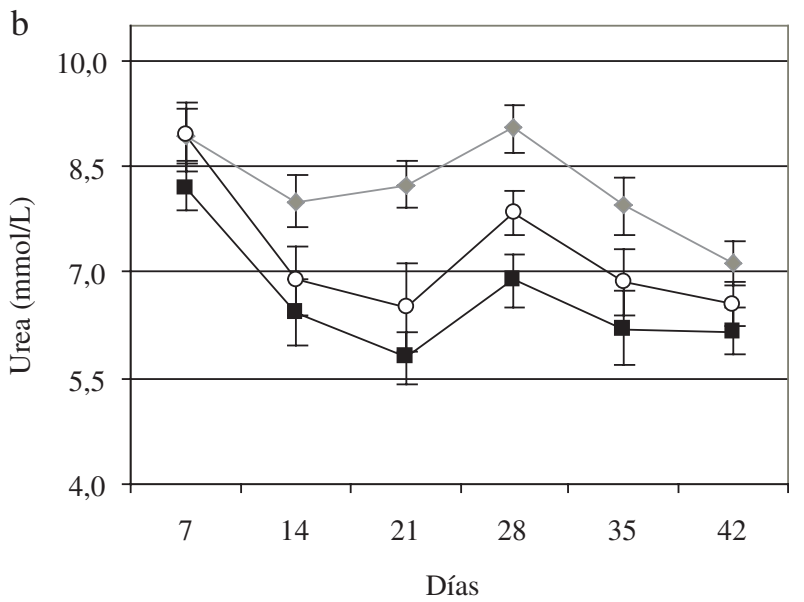

Figura 1. Concentraciones de glucosa (a) y urea (b) plasmática $(\mathrm{X} \pm \mathrm{EE})$ en vacas en lactancia a pastoreo (TP) y suplementadas con concentrado amiláceo (TA) y fibroso (TF) durante un período experimental de 42 días. Diferencia entre períodos, $P<0,05$.

Plasma concentrations of glucose (a) and urea (b) (X \pm SEM) in lactating cows grazing rye grass (TP) and supplemented with concentrate based on starch (TA) or fiber (TF) during 42 days. lo que indicaría un mejor aprovechamiento de la fuente de energía del concentrado amiláceo (cuadro 2). En otro trabajo no se encontraron diferencias en la glucemia de vacas suplementadas con concentrados fibroso y amiláceo (Delahoy y col 2003). Si bien en dicho trabajo el concentrado fibroso utilizado fue elaborado con una mezcla de almidón y fibra digestible provenientes de distintos alimentos, lo cual no ocurrió en este experimento.

Las vacas suplementadas con concentrado amiláceo presentaron concentraciones de $\mathrm{\beta OH}$-butirato inferiores a los tratamientos TP y TF ( $\mathrm{P}<0,05$; cuadro 2$)$, indicando que la suplementación energética con fuentes de rápida degradación ruminal en vacas pastoreando praderas de alta calidad favorecería el balance energético del animal en forma más eficiente que las fuentes de más lenta degradación, como la del concentrado fibroso.

Estudios realizados en vacas de lechería a pastoreo determinaron que la suplementación con concentrado a base de fibra disminuye en un 5,4\% la producción de propionato y aumenta los valores porcentuales de butirato y acetato ruminal comparado con la suplementación con concentrado a base de almidón (Bargo y col 2003). Al incluir en la dieta concentrados amiláceos, en especial el almidón de cebada, aumenta la producción ruminal de propionato, considerado el principal precursor de glucosa en los rumiantes, en perjuicio de butirato y acetoacetato (Knowlton y col 1998). Esta condición mejora el aporte energético al animal, incrementando las concentraciones de glucosa, disminuyendo la movilización de lípidos y con ello la concentración plasmática de $\beta \mathrm{OH}$-butirato; asociado, además, a una menor disponibilidad y absorción de butirato desde el rumen (Miettinen y Huhtanen 1996, Bargo y col 2003).

Al aumentar la concentración ruminal de butirato disminuye la de propionato y consecuentemente las concentraciones de glucosa plasmática y de lactosa en la leche (Huhtanen y col 1993, Miettinen y Huhtanen 1996). Estos efectos estarían asociados a la liberación de insulina, la cual aumentaría la lipogénesis y disminuiría la lipólisis, razón por la cual frente a una reducción del propionato ruminal se reduce la liberación de insulina, incrementando el $\beta \mathrm{OH}$-butirato plasmático (Brockman 1982). El incremento de $\beta \mathrm{OH}$-butirato plasmático llevó a la presentación de cetosis subclínica en algunos de los animales del grupo TP, lo que indica que estos animales presentaron un balance energético negativo.

La cetosis subclínica se presenta habitualmente entre las $2^{\mathrm{a}}$ y $7^{\mathrm{a}}$ semanas posteriores al parto (Nielsen y col 2005 ), por lo que su presentación posterior a la $10^{\mathrm{a}} \mathrm{se}-$ mana de lactancia podría ser atribuida al cambio a la dieta del período experimental, que se inició a los $56 \pm 14$ días de lactancia, la cual llevó a que las vacas del grupo TP dejaran de recibir el concentrado que se les aportó hasta esa fecha. Si bien la cetosis puede desarrollarse durante días o semanas (Nielsen y col 2005), es paradójico observar que el animal que cursó durante el mayor 
período de tiempo con cetosis subclínica fue el que estaba mayor tiempo en lactancia y con menor producción láctea, lo que podría sugerir que su producción estaba afectada por la cetosis (Nielsen y col 2005).

El grupo TA, con excepción del primer muestreo, mantuvo concentraciones plasmáticas de urea dentro de los rangos de referencia, mientras que los grupos TF y TP presentaron valores sobre el rango (>7,0 mmol/L) (Wittwer y col 1999). Las menores concentraciones de urea en las vacas de los tratamientos TA y TF respecto a TP indican un mejor aprovechamiento por parte de las bacterias ruminales del exceso de proteína degradable aportada por la pradera de primavera. Además, como en esta época el forraje de la pradera presentó un bajo contenido de carbohidratos no estructurales (cuadro 1), en las vacas del tratamiento TP se habría sobrepasado la capacidad de utilización del amonio por parte de los microorganismos ruminales, siendo éste absorbido y transformado a urea en el hígado aumentando su concentración plasmática (Galvis y col 2003, Noro y Wittwer 2003).

Por otra parte, las concentraciones de urea plasmática en el grupo TF fueron superiores a las del grupo TA, indicando que la fuente de carbohidratos en el concentrado a base de almidón tuvo una mejor sincronía entre la degradación ruminal de su energía con la de la proteína de la pradera (Sinclair y col 1993, Sinclair y col 2000) que la obtenida con coseta de remolacha. Lo anterior es concordante con la tendencia mostrada en un experimento de pastoreo, donde se comparó maíz chancado y otro concentrado, donde se remplazó parte del maíz chancado por pulpa de remolacha y cascarilla de soya (Delahoy y col 2003). Esos resultados permiten inferir que la suplementación con concentrado amiláceo en vacas a pastoreo propicia una mejor utilización del nitrógeno de la dieta comparado con uno en base a fibra.

Las concentraciones de urea plasmática dentro de un mismo tratamiento cambiaron entre los muestreos, pero presentando una tendencia similar entre los tratamientos (figura 1b), lo que indicaría que la composición de proteína de la pradera cambió durante el experimento, ya que las concentraciones plasmáticas de urea son muy sensibles a los cambios en el aporte proteico de la dieta, incluso a variaciones durante el día (Noro y col 2004).

La concentración de albúmina plasmática fue similar para los tres grupos de vacas, manteniendo sus valores constantes durante el período del ensayo (cuadro 2), encontrándose dentro de los rangos de referencia para la especie ( 29 a $41 \mathrm{~g} / \mathrm{L}$ ), lo que señala que los animales se encontraban en condiciones adecuadas en cuanto al metabolismo de proteínas. Hay que considerar que, como la vida media de la albúmina es de dos semanas, sería necesario un período más largo de ingesta con la nueva dieta para observar modificaciones en su concentración plasmática (Kaneko 1997). Es por ello que en algunos estudios no se han observado cambios en su concentración frente a modificaciones en la dieta (Mena y col 2004).
Los resultados permiten concluir que la suplementación con un concentrado energético, a base de cebada, en mayor medida, y a coseta, en menor grado, favorece el aprovechamiento del nitrógeno de la pradera, disminuyendo la concentración de urea plasmática y el riesgo de presentación de cetosis subclínica en vacas en lactancia a pastoreo.

\section{RESUMEN}

El experimento se desarrolló con el objetivo de determinar y comparar el efecto de la suplementación con concentrados a base de fibra o almidón, sobre el metabolismo energético en vacas lecheras en pastoreo primaveral. Durante un período de 42 días se utilizaron 27 vacas Frisón Negro, seleccionadas por la producción de leche, días posparto, peso vivo, condición corporal y número de lactancias previas, las que fueron asignadas a 3 tratamientos en un diseño completamente al azar. Los tratamientos consistieron en solo pastoreo (TP), pastoreo más $6 \mathrm{~kg} / \mathrm{vaca} / \mathrm{día}$ de un concentrado amiláceo (TA) y pastoreo más $6 \mathrm{~kg} / \mathrm{vaca} /$ día de un concentrado fibroso (TF). El concentrado se entregó en dos raciones de $3 \mathrm{~kg}$ durante las ordeña de la mañana y de la tarde y las vacas fueron manejadas en pastoreo rotativo en franjas sobre una pradera consistente principalmente de gramíneas. De cada animal se obtuvieron 6 muestras de sangre con heparina y $\mathrm{NaF}$, con un lapso de 7 días, para determinar las concentraciones plasmáticas de $\beta \mathrm{OH}$-butirato, urea, albúminas y glucosa. Las vacas del grupo TA presentaron concentraciones de glucosa superiores y de $\beta \mathrm{OH}$-butirato inferiores a las del grupo TP y TF $(\mathrm{P}<0,05)$. El grupo TP presentó un $44 \%$ de cetosis subclínica no observándose casos en TA o TF. Las concentraciones de urea fueron inferiores $(\mathrm{P}<0,05)$ en TA que en TP $(\mathrm{P}<0,05)$. Las concentraciones plasmáticas de albúminas no variaron entre los tratamientos $(\mathrm{P}>0,05)$. Se concluye que la suplementación con concentrado energético amiláceo favorece el balance energético en vacas en lactancia a pastoreo, y que la suplementación con concentrado energético amiláceo o fibroso aumenta el aprovechamiento del nitrógeno de la pradera, disminuyendo la concentración de urea plasmática y el riesgo de presentación de cetosis subclínica.

\section{REFERENCIAS}

Bargo F, LD Muller, JE Delahoy, TW Cassidy. 2002. Milk response to concentrate supplementation of high producing dairy cows grazing at two pasture allowances. J Dairy Sci 85, 1777-92.

Bargo F, LD Muller, ES Kolver, JE Delahoy. 2003. Invited review: production and digestion of supplemented dairy cows on pasture. J Dairy Sci 86, 1-42.

Brockman RP. 1982. Insulin and glucagon responses in plasma to intraportal infusions of propionate and butyrate in sheep (ovis aries). Comparative Biochem Physiol 73A, 237-238.

Carrier J, S Stewart, S Godden, J Fetrow, P Rapnicki. 2004. Evaluation and use of three cowside tests for detection of subclinical ketosis in early postpartum cows. J Dairy Sci 87, 3725-35.

Delahoy JE, LD Muller, F Bargo, TW Cassidy, LA Holden. 2003. Supplemental carbohydrate sources for lactating dairy cows on pasture. J Dairy Sci 86, 906-15.

Galvis R, H Correa, N Ramírez. 2003. Interacciones entre el balance nutricional, los indicadores del metabolismo energético y proteico y las concentraciones plasmáticas de Insulina, e IGF-1 en vacas en lactancia temprana. Rev Col Cs Pec 16, 237-247.

Geishauser T, K Leslie, D Kelton, T Duffield. 2001. Monitoring for subclinical ketosis in dairy herds. Comp Food Anim 23, S65-S71.

Herdt TH. 1988. Fuel homeostasis in the ruminant. Vet Clin North Am: Food Anim Pract 4, 213-231. 
Herdt TH. 2000. Ruminant adaptation to negative energy balance Influences on the etiology of ketosis and fatty liver. Vet Clin North Am: Food Anim Pract 16, 215-230.

Hodgson J. 1990. Grazing management. Science into Practice. Longman Scientific and Technical.

Huhtanen P, H Miettinen, M Ylinen. 1993. Effect of increasing ruminal butyrate on milk yield and blood constituents in dairy cows fed a grass silage-based diet. J Dairy Sci 76, 1114-24.

Kaneko JJ. 1997. Serum proteins and the dysproteinemias. J. J. Kaneko, J. W. Harvey, M. L. Bruss. Clinical Biochemistry of Domestic Animals. Academic Press, San Diego, Pp 117-138.

Knowlton KF, TE Dawson, BP Glenn, GB Huntington, RA Erdman. 1998. Glucose metabolism and milk yield of cows infused abomasally or ruminally with starch. J Dairy Sci 81, 3248-58.

Leaver JD. 1985. Milk production from grazed temperate grassland. J Dairy Res 52, 313-44.

Mena H, JE Santos, JT Huber, M Tarazon, MC Calhoun. 2004. The effects of varying gossypol intake from whole cottonseed and cottonseed meal on lactation and blood parameters in lactating dairy cows. J Dairy Sci 87, 2506-18.

Miettinen H, P Huhtanen. 1996. Effects of the ratio of ruminal propionate to butyrate on milk yield and blood metabolites in dairy cows. J Dairy Sci 79, 851-61.

Nielsen NI, NC Friggens, MG Chagunda, KL Ingvartsen. 2005. Predicting risk of ketosis in dairy cows using in-line measurements of betahydroxybutyrate: a biological model. J Dairy Sci 88, 2441-53.

Noro M, J Borkert, V Vargas, A Hinostroza, R Pulido, F Wittwer. 2004. Diurnal variation in blood metabolites concentration in lactating dairy cows grazing rye pasture. XI Congress international society animal clinical biochemistry-ISACB, Valdivia, Chile, Pp 46.

Noro M, F Wittwer. 2003. Utilidad de la determinación de la urea en la leche. Vetermas 2, 2-5.

Payne J, S Payne. 1987. The Metabolic Profile Test. New York.
Peyraud JL, L Delaby. 2001. Ideal concentrate feeds for grazing dairy cows responses to supplementation in interaction with grazing management and grass quality. P. C. Garnsworthy, J. Wiseman. Recent Advances in Animal Nutrition. Notthingham University Press, UK. Pp 203.

Pulido RG, P Aguilera, R Dae117-138tz, F Wittwer, P Orellana. 2004 Effect of type of concentrate on milk production and composition of dairy cows. J Dairy Sci 87, 224.

Pulido RG, O Balocchi, J Fernandez. 2001. Efecto del nivel de producción de leche sobre el comportamiento ingestivo en vacas lecheras en pastoreo primaveral. Arch Med Vet 33, 137-144.

Pulido RG, M Cerda, W Stehr. 1999. Efecto del nivel y tipo de concentrado sobre el comportamiento productivo de vacas lecheras en pastoreo primaveral. Arch Med Vet 31, 177-187.

Pulido RG, E Felmer, A Hinostroza, F Wittwer. 2004 ${ }^{\text {b }}$. Effect of type of concentrate on grazing behaviour of dairy cows. J Dairy Sci 87, 31 .

Ruiz I. 1997. Conceptos generales del rol de la pradera en la producción de leche. Serie de Simposios y Compendios Sociedad Chilena de Producción Animal, 5, 13-37.

Sinclair KD, LA Sinclair, JJ Robinson. 2000. Nitrogen metabolism and fertility in cattle: I. Adaptative changes in intake and metabolism to diets differing in their rate of energy and nitrogen release in the rumen. J Anim Sci 78, 2659-2669.

Sinclair L, P Garnsworthy, J Newbold, P Buttery. 1993. Effect of synchronizing the rate of dietary energy and nitrogen release on rumen fermentation and microbial protein synthesis in sheep. J Agric Sci 120, 251-263.

Webster AJF. 1993. Understanding the dairy cow. Bodmin, Cornwall, UK. Wittwer F, H Böhmwald, PA Contreras, YJ Filoza. 1987. Análisis de los resultados de perfiles metabólicos obtenidos de rebaños lecheros en Chile. Arch Med Vet 19, 35-45.

Wittwer FG, P Gallardo, J Reyes, H Optiz. 1999. Bulk milk urea concentrations and their relationship with cow fertility in grazing dairy herds in southern Chile. Prev Vet Med 38, 159-66. 